\title{
MODULATION OF OXIDATIVE STRESS BY ABHRAK BHASMA IN DROSOPHILA MELANOGASTER
}

\section{RAMBHADUR P SUBEDI ${ }^{1 *}$, REKHA R VARTAK ${ }^{2}$, PURUSHOTTAM G KALE ${ }^{1}$}

${ }^{1}$ Department of Biological Sciences, Ramniranjan Jhunjhunwala College, Ghatkopar (W), Mumbai - 400 086, Maharashtra, India. ${ }^{2}$ Biology Olympiad Cell, Homi Bhabha Centre for Science Education-TIFR, Mankhurd, Mumbai - 400 088, Maharashtra, India. Email: subedi229@gmail.com

Received: 28 December 2017, Revised and Accepted: 29 January 2018

\begin{abstract}
Objective: Management of aging is one of the most actively researched areas in biology. Ayurvedic preparations such as Abhrak Bhasma (AB) have been used for improving longevity and regenerative therapy as well as in treating various ailments. Modes of action of such Ayurvedic preparations are poorly understood at molecular level. Current investigation tests the efficacy of AB in modulating various parameters linked to oxidative stress.
\end{abstract}

Methods: Drosophila melanogaster was used as a model system to test the efficacy of AB in inducing antioxidant machinery. Drosophila flies were fed on diet supplemented with $\mathrm{AB}$ at larval and adult stages. Subsequently, various parameters, catalytic as well as non-catalytic, related to antioxidant machinery were analyzed.

Results: $\mathrm{AB}$ has been found to modulate the activity of superoxide dismutase and catalase enzymes as well as the total reduced glutathione (GSH) content. Larvae and adults feeding on diet supplemented with AB exhibit significantly lower levels of total GSH content (decrease of about 40-70\% for larvae, while $31-36 \%$ for adults) and without any conclusive effect on GSH:oxidized glutathione ratio, free radical scavenging capacity, and extent of lipid peroxidation. These larvae and adults fed on diet supplemented with AB also exhibited an increase in the level of transcription of cap " $n$ " collar C, heat shock protein 70 , and catalase genes ( $\geq 1.5$ fold, except in few cases).

Conclusion: Overall, $\mathrm{AB}$ alters various parameters linked to antioxidant machinery in D. melanogaster. The induced components may provide protection to the organism during stressful conditions.

Keywords: Lifespan, Ayurvedic, Bhasma, Oxidative stress.

(C) 2018 The Authors. Published by Innovare Academic Sciences Pvt Ltd. This is an open access article under the CC BY license (http://creativecommons. org/licenses/by/4. 0/) DOI: http://dx.doi.org/10.22159/ajpcr.2018.v11i5.24472

\section{INTRODUCTION}

According to the free radical theory of aging, the cells age and die due to the accumulation of non-desirable changes induced by various reactive free radical species. These free radicals such as reactive oxygen species (ROS) are constantly generated in cells of aerobes during normal physiological processes $[1,2]$. To neutralize these ROS, the organisms have an inherent antioxidant system having catalytic as well as noncatalytic components. Studies thus far suggest that a constant battle exists between free radicals and the antioxidant machinery of an organism $[3,4]$. The capacity of an organism to counter the free radicals decreases with age and the accumulation of these damages caused over duration consequently leads to ailments and/or death of an organism.

The non-catalytic machinery of the cell/tissue is the first line of chemical defense against free radicals being generated under normal as well as pathological conditions. Glutathione system, thioredoxin system, and vitamins are some of the major non-catalytic components that target the free radicals in non-specific manner [5-7]. The catalytic antioxidant machinery mainly involves constitutive and/or induced enzyme systems that target specific free radicals. Moreover, various intermediate metabolites in the catalytic pathway have been shown to also act as signaling molecules for managing the stress [8]. Superoxide dismutase (SOD), catalase, and peroxidase are some of the key enzymes of this machinery and show ubiquitous presence in most of the living organisms [9].

It has been a constant endeavor of researchers to modulate the oxidative parameters to delay or counter the effect of oxidative stress and thus increase the lifespan of an organism $[10,11]$. The antioxidant capacity of an organism can be strengthened by employing antioxidant compounds as dietary supplements. These antioxidant supplements may be natural or synthetic in origin. Vitamins that are naturally occurring compounds play a key role in boosting antioxidant capacity, either directly by participating in free radical scavenging or indirectly by promoting the action of other regulators in antioxidant machinery [5,6,12]. Vitamins, in general, have been used for increasing the lifespan in insects [13]. There are, however, contradicting opinions about vitamins such as ascorbic acid (ASC) or Vitamin C, a type of water-soluble vitamin, for enhancing the antioxidant capacity and consequently lifespan in insects $[13,14]$.

Apart from vitamins and other naturally occurring antioxidants, traditional formulations are also presumed to boost antioxidant capacity $[15,16]$. However, a considerable amount of work still needs to be done at the molecular level, to understand the underlying mechanism of action. Abhrak Bhasma (AB) is one such preparation that is produced by repeated incineration of mica with specific medicinal herbs. It is considered as a type of nanomedicine [17] and is often used in the treatment of a variety of ailments, particularly those arising due to oxidative stress [18]. AB is also used as a major component of many rejuvenating or antiaging formulations [15]. However, the mode of action of $\mathrm{AB}$, so far, has not been completely understood.

In the present investigation, an attempt has been made to comprehend the efficacy of $A B$ as a dietary supplement in modulating the oxidative parameters in Drosophila melanogaster. The effect of ASC, a known natural antioxidant, has also been studied for comparison. 


\section{METHODS}

\section{$\mathrm{AB}$}

Sahastraputi $\mathrm{AB}$ that undergoes 1000 cycles of incineration or "puta" was procured from Dhootapapeshwar Ltd., among the leading manufacturers of Ayurvedic medicines (Batch no: P150300110). The detailed characterization of $\mathrm{AB}$ from this source has been performed earlier in our laboratory $[15,19]$.

\section{Drosophila husbandry and test}

D. melanogaster (Canton-S strain) flies were maintained on cornmeal agar medium at $26^{\circ} \mathrm{C}$ under $12 \mathrm{~h}$ light $/ 12 \mathrm{~h}$ dark cycle. Three different sets of experiments were performed to test the efficacy of AB and ASC in modulating oxidative stress parameters at various stages in the life cycle of Drosophila flies. In the first set, the control flies were allowed to lay eggs on cornmeal agar medium containing $0 \% \mathrm{AB}, 0.1 \% \mathrm{AB}, 0.5 \%$ $\mathrm{AB}$, and $20 \mathrm{mM}$ ASC for $6-12 \mathrm{~h}$. The larvae subsequently hatching from these eggs would feed on the respective media. After 5 days of feeding, the $3^{\text {rd }}$ instar larvae were recovered for analysis. In the second set, the larvae raised on Corn Meal Agar medium were allowed to hatch, and the 1-day-old flies were transferred into bottles containing Corn Meal Agar medium with $0 \% \mathrm{AB}, 0.1 \% \mathrm{AB}, 0.5 \% \mathrm{AB}$, and $20 \mathrm{mM}$ ASC. After 7 days, the flies were recovered and further analysis was performed. In the third set of experiment, the control flies were allowed to lay eggs on cornmeal agar medium containing $0 \% \mathrm{AB}, 0.1 \% \mathrm{AB}, 0.5 \% \mathrm{AB}$, and $20 \mathrm{mM}$ ASC for $6-12 \mathrm{~h}$. The larvae subsequently hatching from these eggs would feed on the respective media, and the adult flies emerging from each feeding regime were transferred to separate media bottles containing only Corn Meal Agar (control diet). The flies were recovered at $1,10,20$, and 40 days of adult age for further analysis. This third set of experiment was carried out to trace the effect of feeding larvae with AB-supplemented diet in the adult stages (adults were not fed on diet supplemented with $\mathrm{AB}$ ). For all the subsequent analysis, male and female flies were taken in equal numbers.

\section{Catalase assay}

Catalase assay was performed by monitoring molecular breakdown of $\mathrm{H}_{2} \mathrm{O}_{2}$ by catalase at $240 \mathrm{~nm}$ under $\mathrm{pH} 7$ as per the method described by Aebi [20] and Bai et al. [21]. The larvae as well as adults (20 each) were homogenized in protein extraction buffer containing $20 \mathrm{mM}$ Tris-acetate buffer (pH 7.8), 0.1\% Triton X-100, and $1 \mathrm{mM}$ phenylmethylsulfonyl fluoride using micropestle homogenizer (Sigma cat. no: Z359955-1EA). The tissue extract was then added in $0.1 \mathrm{M}$ phosphate buffer containing $17 \mathrm{mM} \mathrm{H}_{2} \mathrm{O}_{2}$ and its degradation was immediately followed at $240 \mathrm{~nm}$ on Biotek spectrophotometer (Model no: EPOCH-Gen5). Protein content in the tissue extract was measured using Bradford's protein assay method [22]. One unit of catalase activity was defined as the enzyme required for decomposing 1.0 $\mu$ mole of $\mathrm{H}_{2} \mathrm{O}_{2}$ per minute at $\mathrm{pH} 7.0$ at $25^{\circ} \mathrm{C}$ and expressed as enzyme units.

\section{SOD assay}

SOD assay was performed by measuring the inhibition of autoxidation of pyrogallol as described by Marklund and Marklund [23] with slight modifications. The method involves blocking of autoxidation of pyrogallol by SOD. The inhibition of oxidation of pyrogallol was followed at $420 \mathrm{~nm}$ on a Biotek spectrophotometer (Model no: EPOCHGen5). One unit of SOD activity was defined as the amount of enzyme that reduces the pyrogallol autoxidation by $50 \%$ at $\mathrm{pH} 8.5$ at $25^{\circ} \mathrm{C}$ and expressed as enzyme units.

\section{Glutathione content (total reduced glutathione [GSH] and GSH:GSSG ratio)}

The amount of GSH and its oxidized form GSSG were measured using the enzymatic recycling method as described by Rahman et al. [24]. The larvae or adults (20 each) were homogenized in glutathione extraction buffer containing $0.1 \mathrm{M}$ phosphate buffer with 5 mM EDTA (pH 7.5), $6 \mathrm{mg} / \mathrm{ml}$ sulfosalicylic acid, and $0.1 \%$ Triton X-100. Oxidation of GSH was carried out with 5,5'-dithio-bis-(2-nitrobenzoic acid) (DTNB) to form a yellow derivative, 5-thio-2-nitro-benzoic acid (TNB), measurable at $412 \mathrm{~nm}$. The GSSG thus formed is recycled through the activity of glutathione reductase enzyme in the presence of nicotinamide adenine dinucleotide phosphate. The rate of the formation of TNB in comparison to standards was correlated to find the concentration of the GSH and GSSG in samples. The free GSH was derivatized with 2-vinylpyridine for accurate measurement of GSSG. The excess of 2-vinylpyridine was later neutralized with triethanolamine.

\section{2,2-diphenyl-1-picrylhydrazyl (DPPH) scavenging capacity}

The free radical scavenging capacity of the tissue extract was measured using DPPH free radical scavenging assay described by Brand-Williams et al. [25]. Larvae as well as adults (25 each) were homogenized in 0.154 $\mathrm{M} \mathrm{KCl}$ using micropestle homogenizer, and the whole body extract was used for the assay. The varying volumes $(20 \mu \mathrm{l}, 40 \mu \mathrm{l}$, and $100 \mu \mathrm{l})$ of tissue extract were used for DPPH scavenging and measured at $517 \mathrm{~nm}$ on BioTek Spectrophotometer. The amount of tissue required (in terms of $\mathrm{mg}$ of protein) for scavenging DPPH by $50 \%$ was then calculated from the results obtained. Higher the amount of protein required for scavenging $50 \%$ of the DPPH free radicals, lower would be the overall free radical scavenging capacity of the tissue/body.

\section{Lipid peroxidation}

Lipid peroxidation was measured by thiobarbituric acid reactive substances (TBARS) assay method [26,27]. The whole body extracts of larvae/adults (25 each) were homogenized in $\mathrm{KCl}$ and were made to react with thiobarbituric acid (TBA) reagent (containing $0.037 \mathrm{~g}$ TBA, $15 \%$ trichloroacetic acid, and $0.24 \mathrm{~N} \mathrm{HCl}$ per $10 \mathrm{ml}$ ). A reaction mixture of samples as well as different concentration of standard malondialdehyde (MDA) was boiled for $15 \mathrm{~min}$, and the optical density of colored product was measured at $532 \mathrm{~nm}$ on BioTek spectrophotometer. The concentration of MDA in the samples was estimated using the standard MDA plot.

\section{Reverse Transcriptase Polymerase Chain Reaction (RT PCR) and Real-time quantitative PCR (RT-qPCR)}

The total cellular RNA was extracted using TRIZOL method as per manufacturer's instruction (Merck), and subsequently, the cDNA was prepared using SD-prodigy cDNA synthesis kit (SD Fine-chemicals Ltd.). Primers for actin, cap " $\mathrm{n}$ " collar C ( $\mathrm{cncC}$ ), heat shock protein 70 (hsp70), and catalase were designed using the NCBI primer design tool (Table 1).

Amplification of these genes was carried out with the help of PCR using the reaction setting: $94^{\circ} \mathrm{C}$ for 2 min, repeated cycles of 25 cycles: Each of $94^{\circ} \mathrm{C}$ for $30 \mathrm{~s}, 62^{\circ} \mathrm{C}$ for $30 \mathrm{~s}$, and $72^{\circ} \mathrm{C}$ for $30 \mathrm{~s}$ with a final extension at $72^{\circ} \mathrm{C}$ for $5 \mathrm{~min}$. The products thus obtained were then run on $2 \%$ agarose gel. The gel was observed under UV transilluminator, and the images were captured in Uvitec system. The semi-quantitative estimation (RT-PCR) of changes in gene expression was carried out on the captured gel images using "ImageJ" software. Following this, RTqPCR was carried out using SYBR based EVAGREEN (Biorad) on CFX96

Table 1: Genes and primers

\begin{tabular}{llll}
\hline Gene & Product size & Forward & Reverse \\
\hline Actin & $469 \mathrm{bp}$ & CGGCTCGGACAGTGATAGAC & CCGGTACCAAGTATCCTCGC \\
cncC & $147 \mathrm{bp}$ & AGCGCTAGGCTAAAGCAACA & GACAGTTAACGGGACGCTCT \\
hsp 70 & $188 \mathrm{bp}$ & TTGACAACCGGCTAGTCACT & GGTGTAGAAGTCTTGGCCCT \\
Catalase & $366 \mathrm{bp}$ & GAACTGCCCGTACAAGGTGA & GTCAGCATGCGACCGAAATC \\
\hline
\end{tabular}

cncC: Cap "n" collar C, hsp70: Heat shock protein 70 
Real-Time System (Biorad). The cT values were analyzed for fold change in expression of genes using actin as an internal control. Data from both the experiments were compiled, and any mismatch data were repeated for RT-qPCR to obtain accurate results (Triplicates of RT-qPCR were not performed due to the paucity of funds).

\section{Statistical analysis}

Unless specified, all of the experiments were performed in a minimum of three replicates. Statistical significance between any two mean values was analyzed using Student's t-test. For comparing more than two means, analysis of variance (ANOVA) test was performed. ANOVA was immediately followed by Tukey's honest significant difference (HSD) post hoc analysis. Wherever ANOVA showed a significant difference, but Tukey's HSD did not reflect any difference between the groups, Bonferroni and Holm multiple comparison method was applied. Throughout the manuscript, ${ }^{*}$ and ${ }^{* *}$ represent Holms inferences $\mathrm{p}<0.05$ and $\mathrm{p}<0.01$, respectively, while \# and \#\# represent Tukey HSD inference $\mathrm{p}<0.05$ and $\mathrm{p}<0.01$, respectively.

\section{RESULTS}

\section{Larvae versus adults}

\section{Effect of $A B$ on catalytic components}

SOD and catalase enzymes are key components of the catalytic antioxidant system. The activity levels of these enzymes were analyzed after feeding the larvae and adults on media containing two different concentrations of $\mathrm{AB}$. The efficacy of $\mathrm{AB}$ in modulating activity of these enzymes was compared with ASC, a standard antioxidant.

The larvae and adults of Drosophila fed on media containing $0.1 \%$ and $0.5 \% \mathrm{AB}$ exhibited a decrease in SOD activity (ANOVA p value: 0.0296 and 0.0036 , respectively). Feeding of adults on $0.1 \% \mathrm{AB}$ led to about $38 \%$ decrease in SOD activity (Holm inference: $\mathrm{p}<0.01$ ). Whereas, feeding the flies on media containing $0.5 \% \mathrm{AB}$ resulted in a decrease in SOD activity by about $26 \%$ (Holm inference: $\mathrm{p}<0.05$ ) as compared to the adult flies fed on control media. The decrease in SOD activity of adults fed on $0.1 \% \mathrm{AB}$ was also $40 \%$ lower than the adults fed on ASCsupplemented food (Tukey HSD inference: $<0.05$ ) (Fig. 1a). The activity of the SOD enzyme in larvae fed on AB-supplemented diet was not statistically different as compared to the control larvae, but it showed a tendency to decrease due to the feeding of $\mathrm{AB}$.

The larvae fed on $0.1 \%$ and $0.5 \% \mathrm{AB}$ did not show any significant differences in the catalase activity compared to larvae fed on control diet as well as food supplemented with ASC (ANOVA p value: 0.15675). Whereas, the adults fed on $\mathrm{AB}$ resulted in lowering of the catalase activity in comparison to the flies fed on control media (Fig. 1b). A similar decrease in catalase activity was also observed in comparison to the adults fed on a diet supplemented with ASC (ANOVA p value: 0.000168). Feeding the adult flies on ASC-supplemented diet did not alter the catalase activity. However, feeding of flies, at the adult stage, on AB-supplemented diet led to a decrease in catalase activity by about $23 \%$ in $0.1 \% \mathrm{AB}$ (Tukey HSD inference: $<0.01$ ) and $20 \%$ in $0.5 \% \mathrm{AB}$ (Tukey HSD inference: $<0.05$ ) feeding regime. The mean catalase activity of flies fed on media containing $\mathrm{AB}$ was also significantly lower in comparison to the flies fed on media supplemented with ASC (Tukey HSD inference: $<0.01$ for $0.1 \%$ as well as $0.5 \% \mathrm{AB}$ ).

\section{Modulation of non-catalytic components}

The general capacity of $D$. melanogaster to scavenge free radicals was analyzed by measuring the capacity of the whole body extract to scavenge DPPH free radical. Feeding of AB in larval and adult stages resulted in increased amounts of protein required for scavenging DPPH free radicals by about $50 \%$ (Fig. 2). However, this increase was statistically significant only in the larvae fed on AB (ANOVA p value: 0.000874). Feeding of $0.1 \% \mathrm{AB}$ to larvae led to a decrease in the overall free radical scavenging capacity (Tukey HSD inference: $<0.05$ ). This decrease in free radical scavenging capacity was also found to be significantly lower than in the larvae fed on a diet supplemented with ASC (Tukey HSD inference: $<0.01$ ).
Feeding of AB at larvae, as well as adult stages, resulted in a decrease in the total GSH content (ANOVA p value: $6.59^{*} 10^{-7}$ for larvae, 0.0010 for adult). The total GSH content of flies (larvae/adult) from the AB feeding regime was also significantly lower than the flies (larvae/adults) from ASC feeding regime, respectively (Tukey HSD inference: $\mathrm{p}<0.01$ for all comparisons) (Fig. 3a). Between the flies from two different concentrations of $\mathrm{AB}(0.1 \%$ and $0.5 \%)$, no significant differences in the total GSH content of whole body extracts were found. However, larvae fed on $0.1 \%$ and $0.5 \%$ AB-supplemented diet showed an apparent difference in total GSH content. Feeding of $\mathrm{AB}$ at the larval stage exhibited a decrease of about $40 \%$ (for $01 \% \mathrm{AB}$ ) and of $69 \%$ (for $0.5 \%$ $\mathrm{AB}$ ) in total GSH content in comparison to the larvae fed on control media (Holm inference: $\mathrm{p}<0.01$ for both concentrations). The decrease in the total GSH content of the larvae fed on $0.1 \%$ as well as $0.5 \% \mathrm{AB}$ was also significantly different to those larvae fed on ASC-supplemented media (Tukey HSD inference: $\mathrm{p}<0.01$ for both).

GSH: GSSG ratio is another key parameter used as an indicator of oxidative stress. Feeding the larvae as well as adults of $D$. melanogaster on diet supplemented with $\mathrm{AB}$, especially at $0.1 \%$ concentration, showed a tendency to increase in GSH:GSSG ratio. However, this increase was found to be statistically insignificant (overall ANOVA $\mathrm{p}=0.207$ for larvae, while 0.1353 for adults). A similar, statistically insignificant increase was also recorded for larvae and adults fed on ASC-supplemented media (Fig. 3b).

\section{Effect on lipid peroxidation}

The extent of lipid peroxidation was measured as MDA content (TBARS), one of the major products of lipid peroxidation, in tissue/organism. The MDA content in organism increases with age due to the continuous accumulation of reactive oxygen and nitrogen species. Our findings

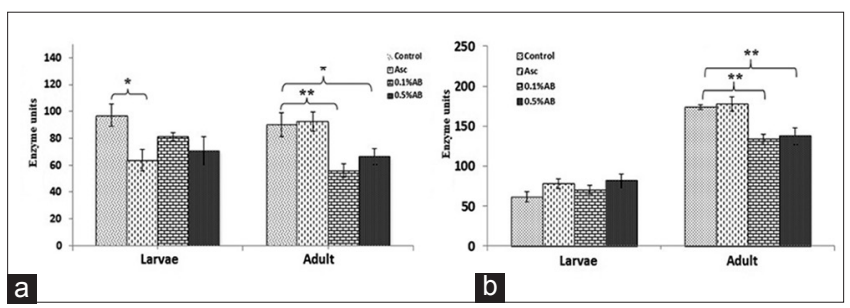

Fig. 1: Effect on catalytic components of larvae and adults. (a). Superoxide dismutase activity. (b). Catalase activity (measured in terms of enzyme units). ${ }^{*}$ and ${ }^{* *}$ represent Holms inferences $\mathrm{p}<0.05$ and $\mathrm{p}<0.01$, respectively

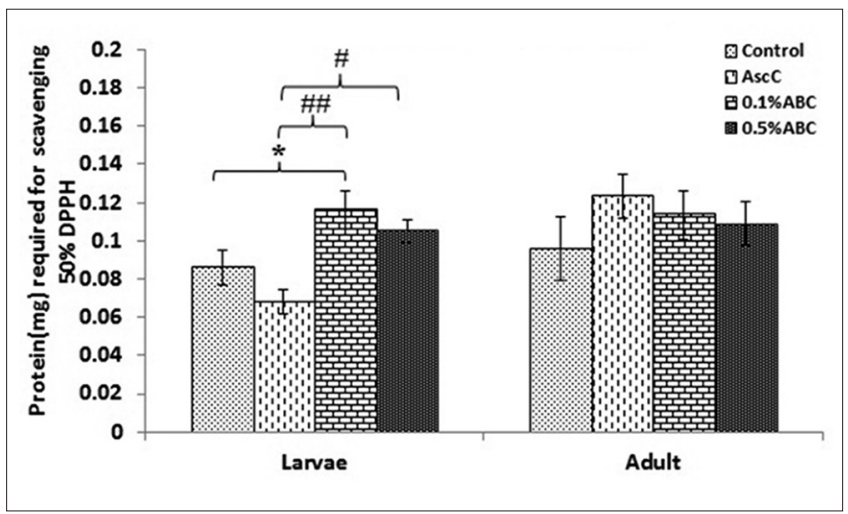

Fig. 2: Effect of dietary antioxidant supplements on free radical scavenging capacity. The capacity to scavenge free radical is measured in terms of $\mathrm{mg}$ of protein (correlates with amount of tissue) required for scavenging $50 \%$ of 2,2-diphenyl-1picrylhydrazyl free radical (higher the amount of tissue required lower is the free radical scavenging capacity). * represents Holms inferences $\mathbf{p}<0.05$, while \# and \#\# represent Tukey honest significant difference inference $p<0.05$ and $p<0.01$, respectively 
were also in accordance with this analogy. However, feeding of larvae as well as adults on the diet spiked with $\mathrm{AB}$ did not cause any significant change in MDA content as compared to the organisms (larvae/adult) fed on the control diet (fig 4). Similarly, feeding of larvae as well as adults on a diet supplemented with ASC did not bring about any significant alteration in the extent of lipid peroxidation (overall ANOVA p value: 0.230688 for larvae groups and 0.20947 for adult groups).

\section{Transcriptional changes}

Feeding of larvae on AB-supplemented media, in general, led to lowering of the level of expression of cncC, hsp70 as well as catalase genes as compared to the expression of these genes in the larvae fed on control media (Fig. 5a). However, the larvae fed on $0.5 \%$ AB feeding regime showed an apparent 1.5-fold increase in the level of catalase gene expression. Contrastingly, feeding the larvae on ASC-supplemented media exhibited $>1.5$-fold increase in the expression of cncC, hsp70, and catalase genes.

Feeding of adults on $\mathrm{AB}$ as well as ASC-supplemented diet, on the other hand, resulted in overexpression of cncC, hsp70, and catalase genes by $\geq 1.5$-fold in comparison to the flies from control media (Fig. $5 \mathrm{~b}$ ). Interestingly, feeding the adults on a diet supplemented with $0.1 \% \mathrm{AB}$ led to an increase by 3 -fold in the level of expression of all these genes. This increase is also greater than the level of expression observed in flies from ASC and $0.5 \% \mathrm{AB}$ feeding regimes.

\section{Effect of larval feeding on adult life}

The larvae fed on media containing $0 \%, 0.1 \%$, and $0.5 \% \mathrm{AB}$ and $20 \mathrm{mM}$ ASC were subsequently allowed to develop into adults. Throughout all their adult life, these flies were maintained on control media without any supplements. All oxidative parameters were followed at the age of $1,10,20$, and 40 days.

The activity of the SOD enzyme was found to increase with age in adult flies from control as well as other feeding regimes (Overall ANOVA $p$ value: $1.5 \times 10^{-6}$ ). Feeding larvae on AB-supplemented diet resulted in a decrease in SOD activity all through the adult life (Fig. 6a). However,

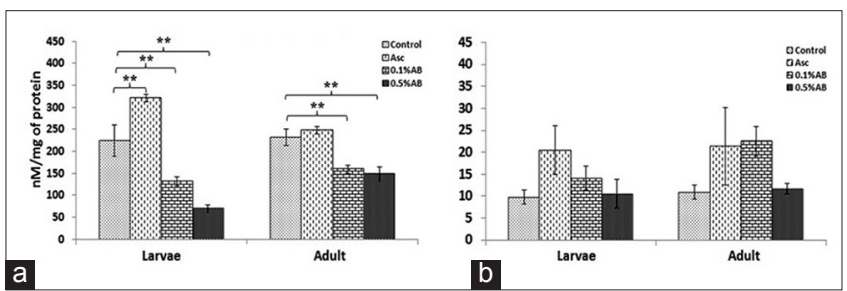

Fig. 3: Effect on non-catalytic components. (a) total reduced glutathione (GSH) content (measured as $\mathrm{nM} / \mathrm{mg}$ of protein). (b) GSH:GSSG ratio ${ }^{* *}$ represents Holms inferences of $\mathbf{p}<0.01$

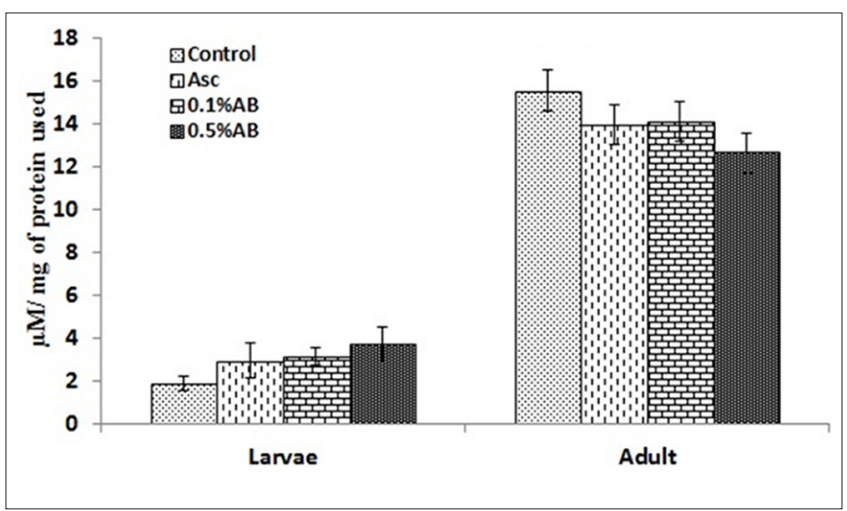

Fig. 4: Effect on lipid peroxidation. The extent of lipid peroxidation was measured as malondialdehyde (MDA) content in the tissue/organism. Higher MDA content is indicative of increase in lipid peroxidation this decrease was only statistically significant in the 10-day-old flies (ANOVA p value: 0.000341 , Tukey HSD inference: $<0.01$ for $0.1 \%$ as well as $0.5 \% \mathrm{AB}$ ). Similarly, at 10 days age, the adults that emerged from larvae fed on ASC-supplemented media and also registered a decrease in SOD activity as compared to the activity noticed in control flies (Tukey HSD inference: <0.05)

In contrast to SOD, the activity of catalase enzyme was found to decrease with age, irrespective of the feeding regime (overall ANOVA: $6.36 \times 10^{-17}$ ). In all age groups, the activity of catalase enzyme in flies from $\mathrm{ASC}$ and $\mathrm{AB}$ feeding regimes remained lower as compared to that recorded in control flies (Fig. 6b). However, no statistically significant difference was observed in the level of catalase activity in 1-day and 20-day-old adults from different feeding regimes (ANOVA $\mathrm{p}$ values: 0.097394 and 0.138468 , respectively). The adults from 10 days of age group under $\mathrm{AB}$ and $\mathrm{ASC}$ feeding regimes showed a significant decrease in catalase activity in comparison to control flies (ANOVA $p$ value: 0.000189 , Tukey HSD inference: $<0.01,<0.01$, and $<0.05$ for $0.1 \% \mathrm{AB}, 0.5 \% \mathrm{AB}$, and $\mathrm{ASC}$, respectively). Whereas from the 40 days old age group, only the adults from $0.5 \% \mathrm{AB}$ feeding regime exhibited a significant decrease in the catalase activity compared to that in control flies (overall ANOVA p value: 0.000103 and Tukey HSD inference: $<0.01$ ). This decrease in catalase activity in adults from $0.5 \% \mathrm{AB}$ feeding regime was also significantly lower than that observed in flies from $0.1 \% \mathrm{AB}$ as well as ASC feeding regime (Tukey HSD inference: $<0.01$ and $<0.01$, respectively).

The free radical scavenging capacity of the whole body extract of the flies, measured in terms of $\mathrm{mg}$ of protein required to scavenge $50 \%$ of the DPPH radicals, did not differ significantly with age (Fig. 7a). Within the different age groups, no significant difference in free radical scavenging capacity was observed, irrespective of the feeding regime with which it is compared (overall ANOVA p value: 0.13306 ).

The extent of lipid peroxidation, measured in terms of MDA content, increased with age (overall ANOVA p value: $4.39 \times 10^{-23}$ ). The whole body extracts of younger flies ( 1 day and 10 days) within specific age groups showed comparable MDA contents, irrespective of the feeding regime

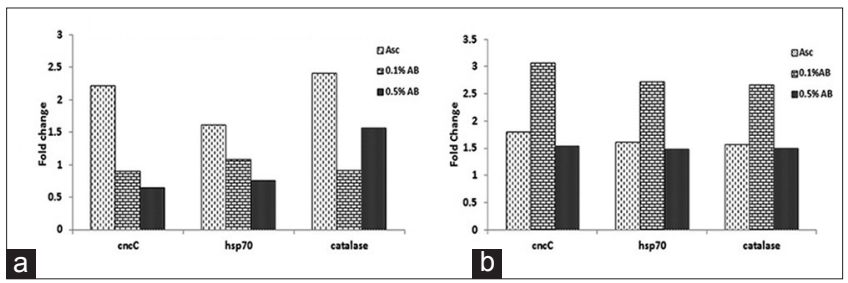

Fig. 5: Effect at transcriptional level in (a) larvae and (b) adult.

The effect of feeding ascorbic acid and abhrak bhasma as a dietary supplement on three major stress induced components, i.e., cap " $n$ " collar $C$, heat shock protein $\mathbf{7 0}$, and catalase gens in Drosophila melanogaster analyzed using real-time polymerase chain reaction. The fold change is calculated considering the expression by control flies (larvae/adult) as baseline, i.e., 1-fold

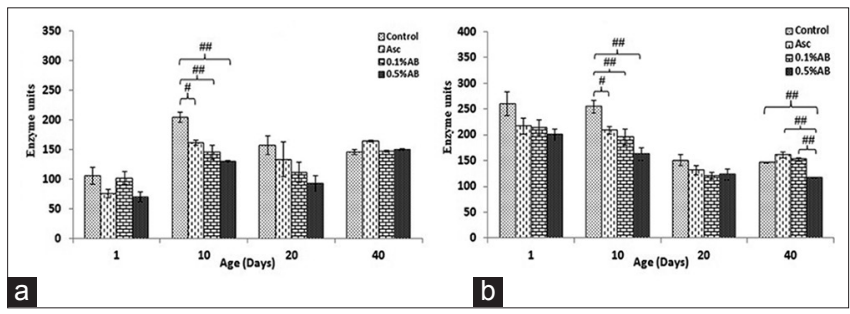

Fig. 6: Larval feeding effect on adult life-catalytic components (a). Superoxide dismutase activity, (b) catalase activity. \# and \#\# represent Tukey honest significant difference inference of $p<0.05$ and $\mathbf{p}<0.01$, respectively 
with which it is compared (ANOVA p value: 0.7211 and 0.53457 for day 1 and 10 days, respectively). While, in relatively older flies from 20 days and 40 days of age groups, flies from the $\mathrm{AB}$ feeding regime (particularly $0.1 \% \mathrm{AB}$ ) exhibited lower MDA content compared to flies from control as well as ASC feeding regimes (ANOVA p value: 0.031935 and $3.8 \times 10^{-5}$, respectively). However, among the flies of 40 days of age group, the total MDA content increased significantly in the flies from $0.5 \% \mathrm{AB}$ as well as ASC feeding regimes as compared to the flies of control feeding regime (Tukey HSD inference: $<0.01$ and $<0.05$, respectively). The MDA content of these flies was also significantly higher than in the flies from $0.1 \% \mathrm{AB}$ feeding regime (Tukey HSD inference: $<0.01$ for both the groups) (Fig. 7b).

The total GSH content in flies from all the feeding regimes was relatively high on their $1^{\text {st }}$ day of emergence as adults (Fig. 8a). The total GSH content dropped to half, by the time, these flies had reached 10 days of adult age (overall ANOVA p value: $1.98 \times 10^{-11}$ ) and remained nearly constant till the maximum lifespan studied (40 days). The total GSH content of the flies from $\mathrm{AB}$ as well as the ASC feeding regime was found to be lower in comparison to that in control flies (ANOVA $p$ value: $0.39155,0.004709$, and 0.002459 for 1 day, 10 days, and 20 days, respectively). This pattern remained constant till the flies attained the 20 days of adult age. In the 40 days of age group, the control flies exhibited lower GSH content in comparison to the GSH content in the flies from other feeding regimes. However, the slight differences observed in the total GSH content of flies, within 1 day and 40 days of age groups, were found to be statistically insignificant (ANOVA p value: 0.64606 for 40 -day-old flies). Within the group, at all ages, the flies from $\mathrm{AB}$ and $\mathrm{ASC}$ feeding regimes did not show any statistically significant difference in the total GSH content among each other.

Similar to the pattern of results obtained for total GSH content, GSH:GSSG ratio also decreased with age in the adult flies (overall ANOVA p value: $7.7 \times 10^{-10}$ ). During the aging from 1 to 10 days, the GSH:GSSG ratio decreased almost by half (Fig. 8b). The differences in GSH:GSSG ratio within various feeding regimes at 1,10 , and 40 days were, however, statistically insignificant (ANOVA p value: 0.15015 , 0.2501 , and 0.38851 , respectively). At 20 days of age, however, the GSH: GSSG ratio was significantly higher in flies from $0.1 \%$ as well as $0.5 \% \mathrm{AB}$ feeding regime in comparison to flies from control feeding regime (ANOVA $p$ value: 0.0334 ).

\section{Effect on the level of transcription}

The cncC, hsp70, and catalase genes are known to be regulators in various stress conditions. Feeding of $\mathrm{AB}$ at the larvae stage resulted in altered expression of cncC, hsp 70, and catalase genes even at the different adult ages (Figs. 9 and 10). Except at the age of 10 days, the flies from the $A B$ feeding regimes showed nearly 2 -fold increase in the expression of $\mathrm{cncC}$ gene in flies of all age groups, as compared to the expression in control flies. The flies from ASC feeding regime, on the other hand, exhibited an increase in transcription of cncC gene at 1 day of adult age in comparison

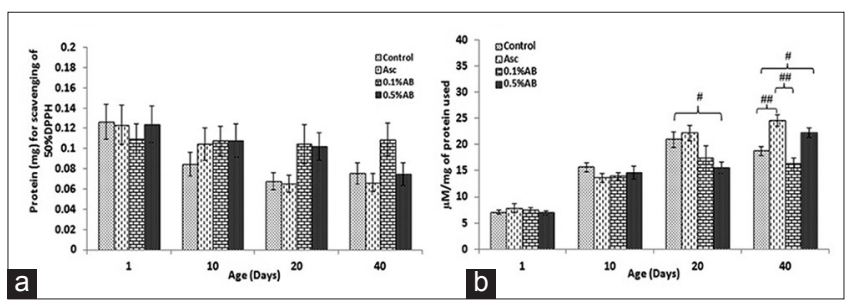

Fig. 7: Larval feeding effect on adult life: (a) Free radical scavenging capacity. It was measured as the amount of protein required (represents the amount of tissue used) for scavenging 2,2-diphenyl-1-picrylhydrazyl free radical by $50 \%$ in vitro. (b) Lipid peroxidation. The extent of lipid peroxidation was measured in terms of total malondialdehyde content in the tissues. \# and \#\# represent Tukey honest significant difference inference of $p<0.05$ and $p<0.01$, respectively to the control flies. However, for other groups, expression of the $\mathrm{cncC}$ remained constant or marginally underexpressed.

The level of expression in the hsp70 gene showed variation with age and also with the type of food regime. In general, the flies from $A B$ feeding regime showed higher levels of hsp70 gene expression $(>1.5$ fold) in most of the age groups. However, at 10 days of age, the level of expression of hsp70 gene remained similar to that encountered in the control flies.

The flies from ASC feeding regime, on the other hand, exhibited about 1.5 -fold increase in the expression of hsp70 gene during 1 and 20 days of age in comparison to the expression noticed in control flies. At 10 days of age, the expression of hsp70 gene remained slightly lower than the expression in control flies, while at 40 days of age, it was equivalent to that found in control flies.

The level of expression of catalase gene in the flies from $A B$ feeding regime remained $\geq 1.5$-fold higher than in control flies during the age of 1,20 , and 40 days. However, at the age of 10 days, expression level of catalase gene was similar in flies from control and $A B$ feeding regimes.

On the contrary, the flies from ASC feeding regime exhibited the expression of catalase gene identical to that in the control flies. In 1-dayold flies, though, the level of expression of catalase gene was nearly half as compared to that in control flies.

In general, the flies from $\mathrm{AB}$ feeding regime maintained a higher level of expression of $\mathrm{cncC}$, hsp70, and catalase genes in comparison to the level of expression in the flies from ASC feeding regime; at nearly all age groups studied.

\section{DISCUSSION}

The results obtained in the present investigation indicate that $A B$, when given as a dietary supplement to Drosophila flies, modulates various parameters related to oxidative stress. For many, if not all, the parameters studied, the effect of $\mathrm{AB}$ has been found comparable to that produced by diet with ASC, a known antioxidant supplement. Some of the key findings from the study can be summarized as follows:

1. Feeding of $A B$ as a dietary supplement at the larval or adult stages leads to decrease in the activity of catalase and SOD enzymes. The larvae fed on $\mathrm{AB}$-supplemented diet when allowed to grow into adults receiving a diet without $A B$ supplement also exhibit the lower activity of catalase and SOD enzymes. These enzymes play a key role in the management of oxidative stress, and a decrease in activity of these enzymes may have negative consequences on organism's life expectancy and physiology. Consumption of $\mathrm{AB}$ was not found to have any adverse effects on the physiology of Drosophila [17]. Thus, $\mathrm{AB}$ plays a role in managing free radicals and compensates for the activity of catalase and SOD enzymes. However, Mitra et al. have shown that Swarna Bhasma, a type of bhasma made with gold, administered to mice results into increase in the activity of catalase as well as SOD enzymes [28].

2. The total GSH content decreases in the larvae and adults fed on diet supplemented with $\mathrm{AB}$. The larvae fed on diet supplemented with $\mathrm{AB}$

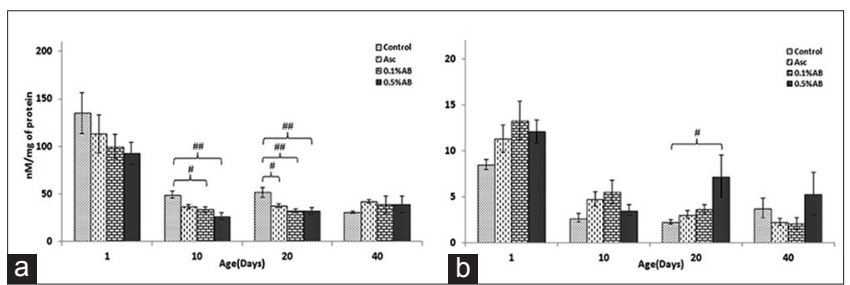

Fig. 8: Effect of larval feeding of test compound on non-catalytic components observed in adult life. (a) Total reduced glutathione (GSH) content. (b) GSH: GSSG ratio. \# and \#\# represent Tukey honest significant difference inference $p<0.05$ and $p<0.01$, respectively 


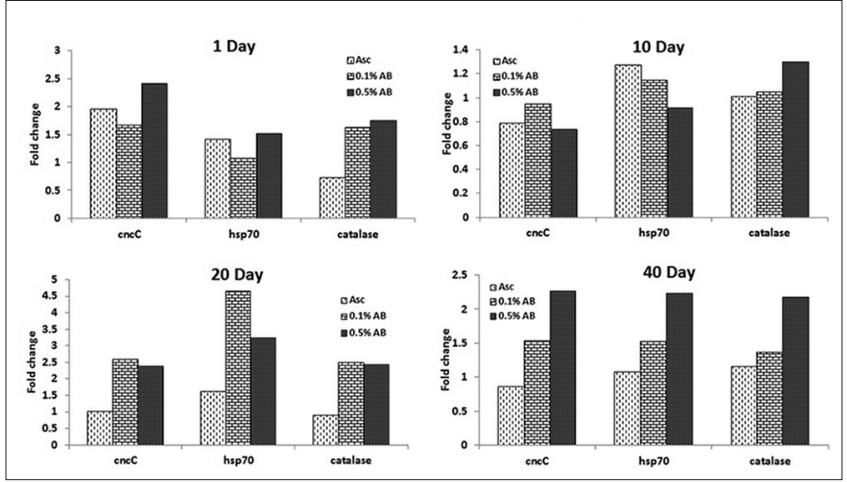

Fig. 9: Effect of feeding test compounds at larval stage on transcription level of stress-induced genes in various stages of adult life (quantified using real-time polymerase chain reaction)

when allowed to develop into adults maintained on normal diet also show a decrease in total GSH content as compared to control flies, throughout the span of 40 days. It has been reported that increase in GSH content leads to a proportionate increase in antioxidant capacity of an organism [29-31]. In the current study, it has been noticed that even with low concentration of GSH, the intrinsic GSH:GSSG ratio remains unaltered in flies from $\mathrm{AB}$-supplemented regimes. In an earlier study [19], we have established that certain component/s of $\mathrm{AB}$ undergo chemical modifications in digestive tract. This implies that some constituent/s of $\mathrm{AB}$ directly, or after modification in GI tract, is/are behaving in a way similar to that of GSH. Alternatively, components of $\mathrm{AB}$ or their modified moieties are influencing the metabolism of Drosophila such that the capacity to relieve oxidative stress is boosted.

3. The dietary supplement of $\mathrm{AB}$ does not induce any change in the overall free radical scavenging capacity as well as GSH:GSSG ratio. This indicates that supplementing Drosophila diet with $\mathrm{AB}$ does not have any adverse effect on the overall capacity of the flies to neutralize free radicals. AB, by itself, does not have any DPPH free radical scavenging activity in vitro (data not shown). The alterations in the DPPH scavenging potential of the tissue, therefore, can be attributed to various parameters induced by $\mathrm{AB}$. ASC, on the contrary, exhibits a strong DPPH scavenging capacity on its own [32]. Thus, some increase in the capacity to scavenge DPPH-free radicals noticed in the flies from ASC feeding regime may be assigned to the traces of ASC that remains in the gut unabsorbed and/or not metabolized by the organism.

4. The lipid peroxidation (measured as MDA level), on the other hand, is the result of accumulating free radicals and is an important indicator of aging. Feeding of AB, at larval as well as adult stage, does not alter the levels of lipid peroxidation. With age, the MDA levels increase as expected and some variations have been observed among the different feeding regimes at early life stages. Although there has been a tendency toward a decrease in lipid peroxidation during some developmental stages, no statistically distinct decrease in the lipid peroxidation has been seen due to feeding on $\mathrm{AB}$. It also suggests that feeding on both $\mathrm{ASC}$ and $\mathrm{AB}$ does not have any negative influence on the lifespan of Drosophila flies.

5. Transcription levels of $\mathrm{cncC}$, hsp70, and catalase genes in larvae fed on diet supplemented with $\mathrm{AB}$ remains similar to control larvae. However, adults raised from the larvae fed on diet supplemented with $\mathrm{AB}$, during older ages, show a prominent increase in the transcription of these genes. In comparison to control, the adult flies fed on diet supplemented with $\mathrm{AB}$ have exhibited a higher expression of $\mathrm{cncC}$, hsp70, and catalase genes, while the expression of these genes in flies from $\mathrm{AB}$-supplemented regimes has been almost identical to the flies from ASC feeding regime. The $\mathrm{cncC}$ helps in the management of oxidative stress by regulating xenobiotic metabolizing enzymes, antioxidants, molecular chaperones, DNA repair enzymes, and antiinflammatory response proteins [33]. ASC has been shown to activate cncC (Nrf2)-mediated signaling system [34,35] and thus protects

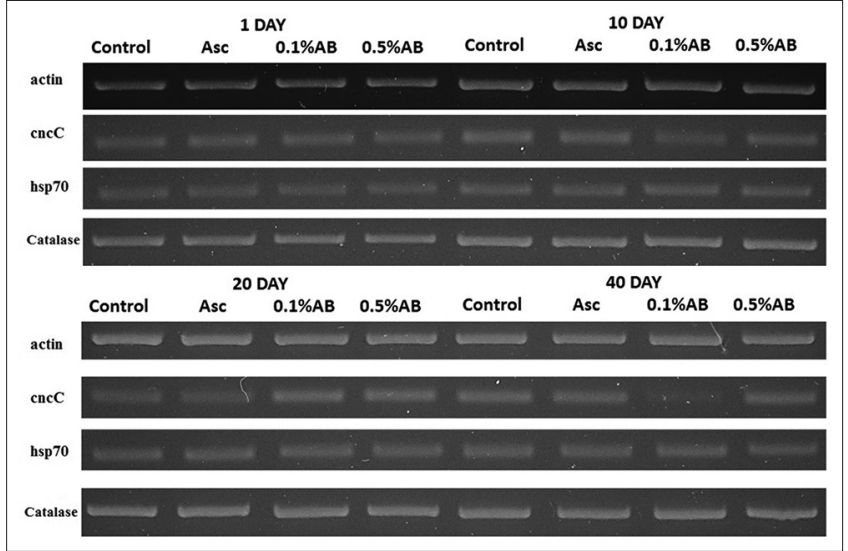

Fig. 10: Effect of feeding test compounds at larval stage on transcription level of stress-induced genes in various stages of adult life (as observed in qualitative real-time polymerase chain reaction)

the organism from harmful effects of oxidative stress. Similarly, the high cncC expression in $\mathrm{AB}$ flies may result in better preparedness for countering the oxidative stress. Similar to $\mathrm{cncC}$, expression of hsp70 gene is also highly regulated during various cellular stresses including oxidative stress [36]. Induction of hsp70 gene expression results in an increase in survival of the organisms due to its protective role as molecular chaperone.

The adults raised from larvae fed on AB and ASC-supplemented diet also show a comparable pattern of expression in the catalase gene. Availability of excessive ASC has been linked with an increase in the hydrogen peroxide formation that may consequently result in cell death [37]. This explains a slight increase in catalase activity as well as increased expression of catalase gene in Drosophila larvae from the ASC feeding regime. However, the adults raised from these larvae show a decrease in catalase activity compared to the control flies at different age points. The larvae that are voracious feeders are likely to be exposed to a greater amount of ASC as compared to the adults; therefore, changes in the catalase activity are bound to be lesser in adults feeding on ASC in comparison to larvae fed on ASC-supplemented food.

Thus, it is evident that $A B$ influences various parameters linked to an antioxidant system of $D$. melanogaster. Earlier [17], we have shown that feeding on $\mathrm{AB}$ improves various physical and behavioral parameters of $D$. melanogaster. The findings in the present investigation, especially at the transcriptional level, strongly supports our earlier propositions. In addition, $\mathrm{AB}$ also seems to induce preadaptation or hormesis-like condition in Drosophila flies. In comparison to the larvae fed on $\mathrm{AB}$, there has been an increase in the expression of cncC, hsp70, and catalase genes during the adult life of larvae fed on $\mathrm{AB}$, even though these adults have not been given $\mathrm{AB}$ supplementation. The lower catalase activity even after increase in transcription of catalase gene can be explained by the fact that transcription and translation can occur at different space and time [38].

We ascribe the variations observed in the effect of $\mathrm{AB}$ and $\mathrm{ASC}$ at larval and adult stages to the differences in the quantity of food consumed. Larvae mainly engulf solid food materials in much larger quantity while adults absorb liquid diet (with or without the test compounds), by suction from the surface of the solid matrix, in relatively small quantity. Therefore, the exposure to the amount of test compounds in the larvae is much higher than in adults. The study strongly supports modulation of various antioxidant parameters due to the feeding of AB. This property is possibly being explored as antiaging formulations and also in the treatment of different diseases.

However, the component/s of $\mathrm{AB}$ that might be playing a key role in the entire process has not been recognized. The preparation of 
$\mathrm{AB}$ or any other traditional formulations faces a serious drawback of complicated procedures for synthesis that may lead to variations in the results. There are no reports on any insect model systems including Drosophila that addresses the mode of action of $\mathrm{AB}$, especially with reference to oxidative stress. Although similar studies have been carried out by Dwivedi et al. in D. melanogaster using Rasa sindoor a type of Bhasma [39], it has been reported that it affects the life history and other physiological traits of D. melanogaster in a positive manner.

\section{CONCLUSION}

Overall, it is evident that supplementation of $A B$ induces some of the parameters related to management of oxidative stress, especially at transcriptional level. It has also been seen that activity or concentration of few catalytic and non-catalytic components decreases due to the feeding of diet supplemented with $\mathrm{AB}$. This, however, does not have any adverse effect on overall free radical scavenging capacity of the organism. Therefore, constituents of AB seem to compensate for decreased activity/ concentration of these components. However, the present investigation should be considered as a preliminary work in this area, and more intense studies are needed before reaching to a definitive conclusion. The future studies may be targeted to enlighten the mode of action of $\mathrm{AB}$, its active ingredients, and efficacy at cellular and molecular levels.

\section{AUTHORS' CONTRIBUTION}

All authors contributed equally in design, execution of experiments, and analysis of the results.

\section{CONFLICTS OF INTERESTS}

Authors express no conflict of interest.

\section{REFERENCES}

1. Sharma P, Jha AB, Dubey RS, Pessarakli M. Reactive oxygen species, oxidative damage, and antioxidative defense mechanism in plants under stressful conditions. J Bot 2012;2012:217037.

2. Lobo V, Patil A, Phatak A, Chandra N. Free radicals, antioxidants and functional foods: Impact on human health. Pharmacogn Rev 2010;4:118-26.

3. Watson J. Oxidants, antioxidants and the current incurability of metastatic cancers. Open Biol 2013;3:120144.

4. Rahal A, Kumar A, Singh V, Yadav B, Tiwari R, Chakraborty S, et al. Oxidative stress, prooxidants, and antioxidants: The interplay. Biol Med Res Int 2014;2014:761264.

5. Meister A. Selective modification of glutathione metabolism. Science 1983;220:472-7.

6. Menni C, Metrustry SJ, Mohney RP, Beevers S, Barratt B, Spector TD, et al. Circulating levels of antioxidant vitamins correlate with better lung function and reduced exposure to ambient pollution. Am J Respir Crit Care Med 2015;191:1203-7.

7. Song M, Kim JS, Liu L, Husain M, Vazquez-Torres A. Antioxidant defense by thioredoxin can occur independently of canonical thioldisulfide oxidoreductase enzymatic activity. Cell Rep 2016;14:2901- 11 .

8. Day RM, Suzuki YJ. Cell proliferation, reactive oxygen and cellular glutathione. Dose Response 2005;3:425-42.

9. Mackay WJ, Bewley GC. The genetics of catalase in Drosophila melanogaster: Isolation and characterization of acatalasemic mutants. Genetics 1989;122:643-52.

10. Pearson KJ, Baur JA, Lewis KN, Peshkin L, Price NL, Labinskyy N, et al. Resveratrol delays age-related deterioration and mimics transcriptional aspects of dietary restriction without extending life span. Cell Metab 2008;8:157-68.

11. Katewa SD, Kapahi P. Dietary restriction and aging. Aging Cell 2010;9:105-12.

12. Gonzalez-Reyes S, Martinez L, Martinez-Calonge W, FernandezDumont V, Tovar JA. Effects of antioxidant vitamins on molecular regulators involved in lung hypoplasia induced by nitrofen. J Pediatr Surg 2006;41:1446-52

13. Pallauf K, Bendall JK, Scheiermann C, Watschinger K, Hoffmann J, Roeder T, et al. Vitamin C and lifespan in model organisms. Food Chem Toxicol 2013;58:255-63.

14. Bahadorani S, Bahadorani P, Phillips JP, Hilliker AJ. The effects of vitamin supplementation on Drosophila life span under normoxia and under oxidative stress. J Gerontol A Biol Sci Med Sci 2008;63:35-42.

15. Bhatia B, Kale PG. Analytical evaluation of an ayurvedic formulationAbhraka Bhasma. Int J Pharm Sci Rev Res 2013;23:17-23.

16. Scartezzini P, Speroni E. Review on some plants of Indian traditional medicine with antioxidant activity. J Ethnopharmacol 2000;71:23-43.

17. Subedi RP, Vartak RR, Kale PG. Effect of abhrak bhasma on the physiology and behaviour of Drosophila melanogaster. Int J Adv Res 2017;5:1629-41.

18. Bhatia B, Kale PG, Daoo JV, Meshram P. Testicular oxidative stress protective effects of Abhrak Bhasma in male Wistar rats after heat exposure. Int J Pharm Pharm Sci 2013;5:472-7.

19. Subedi RP, Vartak RR, Kale PG. Study of general properties of Abhrak Bhasma: A nanomedicine. Int J Pharm Sci Rev Res 2017;44:238-42.

20. Aebi HE. Catalase. In: Method of Enzymatic Analysis. Weinheim, Germany, FL: VCH; 1983. p. 273-86

21. Bai J, Rodriguez AM, Melendez AJ, Cederbaum AI. Overexpression of catalase in cytosolic or mitochondrial compartment protects HepG2 cells against oxidative injury. J Biol Chem 1999;274:26217-24.

22. Bradford MM. A rapid and sensitive method for the quantitation of microgram quantities of protein utilizing the principle of protein-dye binding. Analyt Biochem 1976;72:248-54.

23. Marklund S, Marklund G. Involvement of the superoxide anion radical in the autooxidation of pyrogallol and a convenient assay for superoxide dismutase. Eur J Biochem 1974;47:469-74.

24. Rahman I, Kode A, Biswas SK. Assay for quantitative determination of glutathione and glutathione disulfide levels using enzymatic recycling method. Nat Protocol 2006;1:3159-65.

25. Brand-Williams W, Cuvelier ME, Berset C. Use of free radical method to evaluate antioxidant activity. LWT Food Sci Technol 1995;28:25-30.

26. Zeb A, Ullah F. A simple spectrophotometric method for the determination of thiobarbituric acid reactive substances in fried fast foods. J Ana Meth Chem 2016;2016:9412767.

27. Devasagayam TP, Boloor KK, Ramasarma T. Methods for estimating lipid peroxidation: An analysis of merits and demerits. Indian J Biochem Biophys 2003;40:300-8.

28. Mitra A, Chakraborty S, Auddy B, Tripathi P, Sen S, Saha AV, et al. Evaluation of chemical constituents and free-radical scavenging activity of Swarna Bhasma (gold ash), an ayurvedic drug. J Ethnopharmacol 2002;80:147-53

29. Kerksick C, Willoughby D. The antioxidant role of glutathione and $\mathrm{N}$-acetyl-cysteine supplements and exercise-induced oxidative stress. J Int Soc Sports Nutr 2005;2:38-44

30. Dasari P, Anandamurali R, Nayak P. Effect of tocotrienol pretreatment on ex vivo superoxide and peroxide handling capacities (SPHC) of rat serum and brain. Int J Pharm Pharm Sci 2017;9:116-22.

31. Durga M, Nathiya S, Devasena T. In vitro evaluation of cytotoxicity, oxidative stress, DNA damage and inflammation induced by diesel exhaust particles in human a549 lung cells and murine raw 264.7 macrophages. Int J Pharm Pharm Sci 2014;6:105-10.

32. Janifer R, Chaurasia OP, Vajpayee PK, Pal MM, Singh SB. Antioxidative activity and phytochemical investigation on a high altitude medicinal plant Dracocephalum heterophyllum Benth. Pharmacogn J 2010;2:112-7.

33. Bryan HK, Olayanju A, Goldring CE, Park BK. The Nrf2 cell defence pathway: Keap1-dependent and -independent mechanisms of regulation. Biochem Pharmacol 2013;85:705-17.

34. Kim SR, Ha YM, Kim YM, Park EJ, Kim JW, Park SW, et al. Ascorbic acid reduces HMGB1 secretion in lipopolysaccharide-activated RAW 264.7 cells and improves survival rate in septic mice by activation of Nrf2/HO-1 signals. Biochem Pharmacol 2015;95:279-89.

35. Mikirova N, Scimeca RC. Intravenous high-dose ascorbic acid reduces the expression of inflammatory markers in peripheral mononuclear cells of subjects with metabolic syndrome. J Trans Res 2016;2:188-95.

36. Silver JT, Noble EG. Regulation of survival gene hsp70. Cell Stress Chap 2012;17:1-9.

37. Klingelhoeffer C, Kammerer U, Koospal M, Muhling B, Schneider M, Kapp M, et al. Natural resistance to ascorbic acid induced oxidative stress is mainly mediated by catalase activity in human cancer cells and catalase-silencing sensitizes to oxidative stress. BMC Complement Altern Med 2012;12:61

38. Tian Q, Stepaniants SB, Mao M, Weng L, Feetham MC, Doyle MJ, et al. Integrated genomic and proteomic analyses of gene expression in Mammalian cells. Mol Cell Proteomics 2004;3:960-9.

39. Dwivedi V, Anandan EM, Mony RS, Muraleedharan TS, Valiathan MS, Mutsuddi M, et al. In vivo effects of traditional ayurvedic formulations in Drosophila melanogaster model relate with therapeutic applications. PLoS One 2012; 7:e37113. 